\title{
Global Existence and Temporal Decay of Large Solutions for the Poisson-Nernst-Planck Equations in Low Regularity Spaces
}

\author{
Jihong Zhao ${ }^{1}$ and Xilan $\mathrm{Liu}^{1}$ \\ ${ }^{1}$ Baoji University of Arts and Sciences
}

December 18, 2021

\begin{abstract}
We are concerned with the global existence and decay rates of large solutions for the Poisson-Nernst-Planck equations. Based on careful observation of algebraic structure of the equations and using the weighted Chemin-Lerner type norm, we obtain the global existence and optimal decay rates of large solutions without requiring the summation of initial densities of a negatively and positively charged species is small enough. Moreover, the large solution is obtained for initial data belonging to the low regularity Besov spaces with different regularity and integral indices for the different charged species, which indicates more specific coupling relations between the negatively and positively charged species.
\end{abstract}

\section{Hosted file}

Global existence and decay of solutions.pdf available at https://authorea.com/users/451750/ articles/549938-global-existence-and-temporal-decay-of-large-solutions-for-the-poissonnernst-planck-equations-in-low-regularity-spaces

\section{Hosted file}

Global existence and decay of solutions.tex available at https://authorea.com/users/451750/ articles/549938-global-existence-and-temporal-decay-of-large-solutions-for-the-poissonnernst-planck-equations-in-low-regularity-spaces 